\title{
Comparative occurrence of ischemic stroke with the rhythm versus rate control strategy in a na- tional prospective cohort of atrial fibrillation
}

Jae Guk Kim ${ }^{1,}$, Young Soo Lee 2,, Ki-Woon Kang ${ }^{3}$, Eue-Keun Choi ${ }^{4}$, Myung-Jin Cha ${ }^{4}$, Jung-Myung Lee ${ }^{5}$, Jin-Bae Kim ${ }^{5}$, Junbeom Park ${ }^{6}$, Jin-Kyu Park , Tae-Hoon Kim ${ }^{8}$, Jae-Sun Uhm ${ }^{8}$ Jaemin Shim ${ }^{9}$ Jun Kim ${ }^{10}$, HyungWook Park ${ }^{11}$, Changsoo Kim ${ }^{12}$, and Boyoung Joung ${ }^{8, \dagger}$

\begin{abstract}
${ }^{1}$ Department of Neurology, Eulji University Hospital, Daejeon;

${ }^{2}$ Division of Cardiology, Department of Internal Medicine, Daegu Catholic University Medical Center, Daegu; ${ }^{3}$ Division of Cardiology, Department of Internal Medicine, Eulji University Hospital, Daejeon; ${ }^{4}$ Department of Internal Medicine, Seoul National University Hospital, Seoul; ${ }^{5}$ Division of Cardiology, Department of Internal Medicine, Kyung Hee University Hospital, Seoul; ' ${ }^{6}$ Department of Cardiology, Ewha Womans University School of Medicine, Seoul; ${ }^{7}$ Department of Cardiology, Hanyang University Seoul Hospital, Seoul; ${ }^{8}$ Division of Cardiology, Department of Internal Medicine, Severance Cardiovascular Hospital, Yonsei University College of Medicine, Seoul; ${ }^{9}$ Division of Cardiology, Department of Internal Medicine, Korea University Medical Center, Seoul; ${ }^{10}$ Heart Institute, Asan Medical Center, University of Ulsan College of Medicine, Seoul; ${ }^{11}$ Department of Cardiology, Chonnam National University Hospital, Chonnam National University School of Medicine, Gwangju; ${ }^{12}$ Department of Preventive Medicine, Yonsei University College of Medicine, Seoul, Korea
\end{abstract}

Received: February 16, 2019

Revised : May 5, 2019

Accepted: June 13, 2019
Background/Aims: Comparative occurrence of ischemic stroke for rhythm versus rate control strategy in patients with non-valvular atrial fibrillation (NVAF) is still inconclusive. The purpose of this study was to investigate whether the rhythm control strategy is associated with a lower risk of ischemic stroke compared to the rate control strategy in NVAF patients.

Methods: The CODE-AF registry prospectively enrolled 6,280 consecutive patients who were treated for NVAF at 10 tertiary referral centers in South Korea. Of these, 2,513 NVAF patients (age, $67 \pm 10$ years; male, 61.8\%) were clinically followed up for over 1-year and divided into rate and rhythm control groups.

Results: Those treated with the rhythm control strategy were younger and had less proportions of underlying disease compared to those treated with the rate control strategy. After the propensity matching analysis, those treated with the rhythm control strategy had similar baseline characteristics including the $\mathrm{CHA}_{2} \mathrm{DS}_{2}$-VASC score compared to those treated with the rate control strategy. The rate of oral anticoagulation, all bleeding, and hospitalization were also similarly between the two groups. The incidence rate of ischemic stroke in the rhythm control group was significantly lower than in the rate control group (0.7 vs. 6.9 per 1,000 person-years, $p=0.011$ ).

Conclusions: The rhythm control strategy demonstrated a beneficial effect to lower the risk of ischemic stroke during a 1-year follow-up compared to the rate control strategy.

Keywords: Atrial fibrillation; Rhythm; Rate; Stroke

\section{Correspondence to Ki-Woon Kang, M.D. Dunsanseo-ro, Seo-gu, Daejeon 35233, Korea \\ https://orcid.org/0000-0002-1361-0022 \\ *These authors contributed equally to this work. \\ †Principal Investigator for CODE-AF Registry.}

Division of Cardiology, Department of Internal Medicine, Eulji University Hospital, 95

Tel: +82-42-611-3081, Fax: +82-42-611-3083, E-mail: kwkang@eulji.ac.kr 


\section{INTRODUCTION}

Atrial fibrillation (AF) is a well-known malignant supraventricular arrhythmia, which mainly is associated with the occurrence of ischemic stroke [1,2]. Oral anticoagulation (OAC) based on the $\mathrm{CHA}_{2} \mathrm{DS}_{2}$-VASc score is the only recommended treatment option for the prevention of ischemic stroke in patients with non-valvular atrial fibrillation (NVAF) [2-5]. Recent studies have reported that a restored and maintained sinus rhythm could be another option to reduce the risk of future ischemic stroke [6-8]. However, the rhythm control strategy to lower the risk of ischemic stroke has not yet been proven in patients with NVAF [9-13]. A few studies have shown that the rhythm control strategy is associated with favorable outcomes and a significantly lower risk of stroke $[7,14]$. Therefore, we investigated whether the rhythm control strategy prevents the occurrence of ischemic stroke compared with the rate control strategy in patients with NVAF in a prospective national cohort for at least a 1-year follow-up period.

\section{METHOD}

\section{Study populations}

The COmparison study of Drugs for symptom control and complication prEvention of Atrial Fibrillation (CODE-AF) is a prospective, multicenter and observational study of patients over 18 years of age with NVAF consecutively enrolled in 10 tertiary referral centers which include all geographical regions of South Korea. The study design and follow-up data were previously described [15]. The study was approved by the Eulji University Hospital Institutional Review Board (IRB Number: 2016-05-003), and all the patients provided informed consent for their inclusion. This study was registered at ClinicalTrials.gov (NCTo2786095). The data entered at each center were audited regularly, and the database used for this analysis went through a data cleansing process. The collected data were registered and adjusted in the web-based clinical research management system, iCreat (Internet based Clinical Research and Trial management system, http://icreat.nih. go.kr), provided by the government. From June 2016 to September 2017, a total of 6,280 NVAF patients were prospectively, consecutively enrolled between all the centers. All the enrolled patients were exclusively classified based on the AF treatment strategy (rhythm control versus rate control) selected by the physicians. From the enrolled population, 2,513 patients (40.0\%) were clinically followed up for at least 1-year which consisted of 1,233 patients (49.1\%) treated with the rhythm control strategy and 1,280 patients (50.9\%) treated with the rate control strategy (Supplementary Fig. 1).

\section{Definition of the rate control and rhythm control strategy}

The rate control strategy was defined as using only rate-control achieved by the $\beta$-blockers digoxin and diltiazem, or verapamil and included previous unsuccessful electrical cardioversion (ECV) and atrial fibrillation catheter ablation (AFCA). The rhythm control strategy was defined as using anti-arrhythmic drugs or having received additional non-pharmacological treatments to control, restore and maintain the sinus rhythm which included previous successful ECV and AFCA at enrollment $[2,3,15,16]$.

\section{Definition and assessment of ischemic stroke, bleeding and renal function}

The $\mathrm{CHA}_{2} \mathrm{DS}_{2}$-VASc scores and HAS-BLED (Hypertension, Abnormal renal/liver function, Stroke, Bleeding history or predisposition, Labile international normalized ratio, Elderly, and Drugs/alcohol concomitantly) scores were calculated for all the enrolled patients with NVAF. Ischemic stroke was defined according to the World Health Organization criteria or the Korean Clinical Practice Guidelines for Stroke as rapidly developing signs of focal or global neurologic deficit with cerebral dysfunction without apparent vascular cause $[17,18]$. Chronic kidney disease (CKD) was defined as a glomerular filtration rate less than $60 \mathrm{~mL} / \mathrm{min} / 1.73 \mathrm{~m}^{2}$ according to the Chronic Kidney Disease Epidemiology Collaboration [19]. In patients with NVAF based on the $\mathrm{CHA}_{2} \mathrm{DS}_{2}$-VASc score, HAS-BLED and renal function, OAC was the only mandatory prescription according to the 2016 European Society of Cardiology and 2014 American Heart Association/American College of Cardiology/Heart Rhythm Society guidelines for patients with $\mathrm{AF}[2,20]$. 


\section{Assessment of clinical outcome and follow-up}

The primary outcome was defined as ischemic stroke, and secondary outcomes were defined as all bleeding, all-cause death and all-cause hospitalization. All patients were followed up at the outpatient clinic at 6 and 12 months by monitoring for symptoms and electrocardiogram (ECG). but patients who reported any symptoms suggestive of arrhythmia recurrence had an additional visit to obtain an additional ECG and Holter monitor.

A blinded cardiologist and stroke expert (neurologist) reviewed and assessed the brain magnetic resonance images and clinical outcomes during the 1-year follow-up between the rhythm control and rate control strategies. Major bleeding was defined as fatal bleeding, symptomatic bleeding in a critical area or organ, and bleeding causing a decline in the hemoglobin level of $2 \mathrm{~g} / \mathrm{dL}$ or more or leading to a transfusion of two or more units of whole blood or red cells [21]. Non-major bleeding was defined as any sign or symptom of hemorrhage, including bleeding found by imaging alone, which does not fit the criteria for major bleeding [22].

\section{Statistical analysis}

Continuous variables are expressed as the mean \pm standard deviation, and categorical variables are reported as frequencies or percentages. In addition, propensity score matching was analyzed to reduce the biases of the baseline characteristics between the rate-control and rhythm-control groups [23]. We created a propensity-score-matched dataset by attempting to match each participant who received the rhythm control strategy with one who received the rate control strategy (a 1:1 match). A nearest neighbor-matching algorithm with a "greedy" heuristic (one that always implements the best immediate, local solution) was used to match the participants. In the greedy nearest-neighbor matching, a participant receiving the rhythm control strategy was randomly selected, and the matching was attempted with the "nearest" participant in the rate control group. The differences in the characteristics between the rhythm control group and the rate control group for all the participants were analyzed by independent t-test for continuous variables and chi-square test for categorical variables. Furthermore, to detect the association between the two groups and hospitalization during the long-term follow-up, we used the Kaplan-Meier curve and log-rank test. All statistical analyses were performed using SAS version 9.3 (SAS Inc., Cary, NC, USA) and MedCalc software version 12.3 (Acacialaan, Ostend, Belgium). A $p<0.05$ was considered statistically significant.

\section{RESULTS}

There appeared to be no significant difference in the baseline characteristics between loss of follow-up and at least 1-year follow-up (Supplementary Table 1). In Table 1 , before the propensity score matching, the patients with the rate control strategy were older and had higher proportions of females, hypertension, large left atrium, diabetes, heart failure, and CKD compared to those treated with the rhythm control strategy. In addition, the $\mathrm{CHA}_{2} \mathrm{DS}_{2}$-VASC and HAS-BLED scores for patients treated with the rate control strategy were higher than for those treated with the rhythm control strategy (3.1 \pm 1.6 vs. $2.3 \pm 1.5, p<0.001 ; 2.1 \pm 1.0$ vs. $1.7 \pm 1.0, p<0.001)$. After the propensity score matching shown in Table 2, patients with the rate control strategy were similar in age and had similar proportions of gender, hypertension, diabetes, heart failure, and CKD compared to those treated with the rhythm control strategy except for those with AF in the baseline. The $\mathrm{CHA}_{2} \mathrm{DS}_{2}$-VASC and HASBLED scores for patients treated with the rate control strategy were also similar compared to those treated with the rhythm control strategy.

The majority of the analyzed patients were treated with one or more antithrombotic agents. After the propensity score matching, direct OAC (71.9\% vs. 76.1\%), anti-platelet agents and statins were similarly prescribed in both groups. In the rate control strategy group, $\beta$-blockers were the most commonly prescribed medication followed by diltiazem. In the rhythm control strategy group, flecainide was the most commonly prescribed medication followed by propafenone. Overall, $13 \%$ of the rhythm control group underwent AFCA during the follow-up period shown in Table 3.

The mean follow-up durations $(17.0 \pm 2.2$ months vs. 17.1 \pm 1.9 months) were similar between the rhythm control and rate control groups. The overall event rate of ischemic stroke in the rhythm control group was significantly lower than that of the rate control group (0.1\% 
Kim JG, et al. AF rhythm control strategy for stroke

Table 1. Comparison of the baseline characteristics of patients treated with the rhythm and the rate control strategy

\begin{tabular}{|c|c|c|c|c|}
\hline Characteristic & $\operatorname{ALL}(\mathrm{n}=2,513)$ & Rate control $(\mathrm{n}=1,233)$ & Rhythm control $(\mathrm{n}=1,280)$ & $p$ value \\
\hline Age, yr & $67 \pm 10$ & $70 \pm 9$ & $65 \pm 10$ & $<0.001^{\mathrm{a}}$ \\
\hline Male sex & $1553(61.8)$ & $736(59.6)$ & $817(63.8)$ & $0.032^{\mathrm{a}}$ \\
\hline $\mathrm{BMI}, \mathrm{kg} / \mathrm{m}^{2}$ & & $24.6 \pm 3.5$ & $24.6 \pm 3.0$ & 0.836 \\
\hline Hypertension & $1,759(70.0)$ & $928(75 \cdot 3)$ & $831(64.9)$ & $<0.001^{\mathrm{a}}$ \\
\hline Diabetes mellitus & $679(27.0)$ & $386(31.3)$ & $293(29 \cdot 3)$ & $<0.001^{\mathrm{a}}$ \\
\hline Dyslipidemia & $874(34.8)$ & $452(36.7)$ & $422(33.0)$ & 0.151 \\
\hline Previous MI & $106(4.2)$ & $61(4.9)$ & $45(3 \cdot 5)$ & 0.121 \\
\hline Previous HF & $241(9.6)$ & $152(12.3)$ & $89(7.0)$ & $<0.001^{\mathrm{a}}$ \\
\hline Previous CKD & $234(9.3)$ & $156(12.7)$ & $78(6.1)$ & $<0.001^{\mathrm{a}}$ \\
\hline Previous ICD & $29(1.2)$ & $16(1.3)$ & $13(1.0)$ & 0.283 \\
\hline Previous PPM & $170(6.8)$ & $87(7 \cdot 1)$ & $83(6.5)$ & 0.568 \\
\hline Previous cancer & $238(9.5)$ & $129(10.5)$ & $\log (8.5)$ & 0.096 \\
\hline Previous stroke or TIA & $408(16.2)$ & $210(17.0)$ & $198(15 \cdot 5)$ & 0.288 \\
\hline Previous ECV & $446(7 \cdot 7)$ & $167(13.5)$ & $279(21.8)$ & $<0.001^{\mathrm{a}}$ \\
\hline Previous AFCA & $422(16.8)$ & $144(11.7)$ & $278(21.7)$ & $<0.001^{\mathrm{a}}$ \\
\hline $\mathrm{CHA}_{2} \mathrm{DS}_{2}$-VASC score & & $3.1 \pm 1.6$ & $2.3 \pm 1.5$ & $<0.001^{\mathrm{a}}$ \\
\hline 0 & $62(2.4)$ & $18(1.5)$ & $44(3.4)$ & \\
\hline 1 & $160(6.3)$ & $55(4 \cdot 4)$ & $105(8.2)$ & \\
\hline 2 & $439(17 \cdot 4)$ & $202(16.3)$ & $237(18.5)$ & \\
\hline 3 & $472(18.7)$ & $242(19.6)$ & $230(17.9)$ & \\
\hline 4 & $353(14.0)$ & $220(17.8)$ & $133(10.3)$ & \\
\hline 5 & $228(9.0)$ & $142(11.5)$ & $86(6.7)$ & \\
\hline 6 & $89(3.5)$ & $60(4.8)$ & $29(2.2)$ & \\
\hline 7 & $30(1.1)$ & $25(2.0)$ & $5(0.3)$ & \\
\hline $8-10$ & $8(0.3)$ & $5(0.4)$ & $3(0.2)$ & \\
\hline HAS-BLED score & $1.8 \pm 1.0$ & $2.1 \pm 1.0$ & $1.7 \pm 1.0$ & $<0.001^{\mathrm{a}}$ \\
\hline Systolic BP, mmHg & $121 \pm 15$ & $120 \pm 14$ & $121 \pm 14$ & 0.256 \\
\hline Diastolic BP, mmHg & $73 \pm 11$ & $73 \pm 11$ & $73 \pm 10$ & 0.107 \\
\hline Heart rate, bpm & $74 \pm 15$ & $77 \pm 15$ & $72 \pm 15$ & $<0.001^{\mathrm{a}}$ \\
\hline Ejection fraction, \% & $61 \pm 7$ & $61 \pm 18$ & $62 \pm 8$ & 0.120 \\
\hline LA size, $\mathrm{mm}$ & $43 \pm 8$ & $45 \pm 8$ & $41 \pm 7$ & $<0.001^{\mathrm{a}}$ \\
\hline Baseline AF & $1,170(46.5)$ & $872(71.3)$ & $298(23.2)$ & $<0.001^{\mathrm{a}}$ \\
\hline Symptomatic AF & $1,055(42.0)$ & $529(42.9)$ & $526(41.1)$ & 0.274 \\
\hline AF type & & & & 0.274 \\
\hline Paroxysmal AF & $1,055(42.0)$ & $529(42.9)$ & $526(41.1)$ & \\
\hline Persistent AF & $1,456(57 \cdot 9)$ & $702(56.9)$ & $754(58.9)$ & \\
\hline Permanent AF & $2(0.1)$ & $2(0.2)$ & o & \\
\hline
\end{tabular}

Values are presented as mean \pm standard deviation or number (\%).

BMI, body mass index; MI, myocardial infarction; HF, heart failure; CKD, chronic kidney disease; ICD, intracardiac defibrillator; PPM, permanent pacemaker; TIA, transient ischemic attack; ECV, electrical cardioversion; AFCA, atrial fibrillation catheter ablation; HAS-BLED, Hypertension, Abnormal renal/liver function, Stroke, Bleeding history or predisposition, Labile international normalized ratio, Elderly, and Drugs/alcohol concomitantly; BP, blood pressure; LA, left atrium; AF, atrial fibrillation.

${ }^{\mathrm{a}}$ Statistically significant. 
Table 2. After propensity score matching, comparison of the baseline characteristics of patients treated with the rhythm and the rate control strategy

\begin{tabular}{|c|c|c|c|c|}
\hline Characteristic & $\operatorname{ALL}(n=1,800)$ & Rate control $(\mathrm{n}=900)$ & Rhythm control $(\mathrm{n}=900)$ & $p$ value \\
\hline Age, yr & $67 \pm 9$ & $67 \pm 9$ & $67 \pm 9$ & 0.998 \\
\hline Male sex & $1118(62.3)$ & $558(62.2)$ & $560(62.4)$ & 0.922 \\
\hline BMI, $\mathrm{kg} / \mathrm{m}^{2}$ & & & & $0.010^{\mathrm{a}}$ \\
\hline Normal (18.5-24.5) & $954(53.1)$ & $446(46.7)$ & $508(56.6)$ & \\
\hline Overweight (25-29.9) & $689(38.4)$ & $361(40.2)$ & $328(36.5)$ & \\
\hline Obese $(>30)$ & $107(5.96)$ & $64(7.1)$ & $43(4.79)$ & \\
\hline Hypertension & $1282(71.2)$ & $649(72.1)$ & $633(70.3)$ & 0.442 \\
\hline Diabetes mellitus & $476(26.4)$ & $235(26.1)$ & $241(26.8)$ & 0.352 \\
\hline Dyslipidemia & $639(35 \cdot 5)$ & $323(35 \cdot 9)$ & $316(35.1)$ & 0.942 \\
\hline Previous MI & $70(3.9)$ & $35(3.9)$ & $35(3.9)$ & 0.606 \\
\hline Previous HF & $133(7 \cdot 4)$ & $66(7 \cdot 3)$ & $67(7.4)$ & 0.996 \\
\hline Previous CKD & $131(7 \cdot 3)$ & $63(7.0)$ & $68(7.6)$ & 0.333 \\
\hline Previous ICD & $22(1.2)$ & $11(1.2)$ & $11(1.2)$ & 0.367 \\
\hline Previous PPM & $104(5.8)$ & $49(5.4)$ & $55(6.1)$ & 0.544 \\
\hline Previous cancer & $174(9.7)$ & $90(10.0)$ & $84(9 \cdot 3)$ & 0.632 \\
\hline Previous stroke or TIA & $294(16.3)$ & $136(15.1)$ & $158(17.6)$ & 0.161 \\
\hline Previous ECV & $320(17.8)$ & $143(15.9)$ & $177(19.7)$ & 0.083 \\
\hline Previous AFCA & $309(17.2)$ & $122(13 \cdot 6)$ & $187(20.8)$ & $<0.001^{\mathrm{a}}$ \\
\hline $\mathrm{CHA}_{2} \mathrm{DS}_{2}$-VASC score & $2.7 \pm 1.5$ & $2.7 \pm 1.5$ & $2.7 \pm 1.5$ & 0.542 \\
\hline o & $117(7.6)$ & $53(6.6)$ & $64(8.7)$ & \\
\hline 1 & $216(14.1)$ & $107(13 \cdot 3)$ & $109(14.9)$ & \\
\hline 2 & $369(24.1)$ & $210(26.2)$ & $159(21.7)$ & \\
\hline 3 & $368(24.0)$ & $184(22.9)$ & $184(25.1)$ & \\
\hline 4 & $248(16.2)$ & $137(17.1)$ & $111(15.2)$ & \\
\hline 5 & $141(9.2)$ & $66(8.2)$ & $75(10.2)$ & \\
\hline 6 & $57(3.7)$ & $32(4.0)$ & $25(3.4)$ & \\
\hline 7 & $14(0.9)$ & $10(1.2)$ & $4(0.5)$ & \\
\hline $8-10$ & $4(0.3)$ & $3(0.3)$ & $1(0.1)$ & \\
\hline HAS-BLED score & $2.0 \pm 1.0$ & $2.0 \pm 1.0$ & $2.0 \pm 1.0$ & 0.702 \\
\hline Systolic BP, mmHg & $120 \pm 14$ & $120 \pm 14$ & $121 \pm 14$ & 0.256 \\
\hline Diastolic BP, mmHg & $73 \pm 10$ & $73 \pm 11$ & $73 \pm 10$ & 0.107 \\
\hline Heart rate, bpm & $75 \pm 15$ & $78 \pm 15$ & $72 \pm 15$ & $<0.001^{\mathrm{a}}$ \\
\hline Ejection fraction, \% & $62 \pm 15$ & $61 \pm 20$ & $62 \pm 9$ & 0.697 \\
\hline LA size, $\mathrm{mm}$ & $43 \pm 7$ & $44 \pm 7$ & $42 \pm 6$ & $<0.001^{\mathrm{a}}$ \\
\hline Baseline AF & $838(46.5)$ & $613(68.1)$ & $225(25.0)$ & $<0.001^{\mathrm{a}}$ \\
\hline Symptomatic AF & $1042(57.9)$ & $518(57.6)$ & $524(58.2)$ & 0.152 \\
\hline AF type & & & & $<0.001^{\mathrm{a}}$ \\
\hline Paroxysmal AF & $1283(71.3)$ & $574(63.4)$ & $709(78.7)$ & \\
\hline Persistent AF & $484(26.8)$ & $293(32.5)$ & $191(21.2)$ & \\
\hline Permanent AF & $33(1.8)$ & $33(3.6)$ & 0 & \\
\hline
\end{tabular}

Values are presented as mean \pm standard deviation or number (\%).

BMI, body mass index; MI, myocardial infarction; HF, heart failure; CKD, chronic kidney disease; ICD, intracardiac defibrillator; PPM, permanent pacemaker; TIA, transient ischemic attack; ECV, electrical cardioversion; AFCA, atrial fibrillation catheter ablation; HAS-BLED, Hypertension, Abnormal renal/liver function, Stroke, Bleeding history or predisposition, Labile international normalized ratio, Elderly, and Drugs/alcohol concomitantly; BP, blood pressure; LA, left atrium; AF, atrial fibrillation.

${ }^{\mathrm{a}}$ Statistically significant. 
Table 3. After propensity score matching, comparison of treatments in the rhythm and the rate control strategy groups

\begin{tabular}{|c|c|c|c|c|}
\hline Variable & $\operatorname{ALL}(n=1,800)$ & Rate control $(n=900)$ & Rhythm control ( $\mathrm{n}=900)$ & $p$ value \\
\hline Warfarin & $350(25.9)$ & $190(27.9)$ & $160(23.9)$ & 0.187 \\
\hline Dabigatran & $320(23.7)$ & $144(21.1)$ & $176(26.3)$ & \\
\hline Apixaban & $260(19 \cdot 3)$ & $137(20.1)$ & $123(18.4)$ & \\
\hline Ribaroxaban & $357(26.4)$ & $179(26.3)$ & $178(26.6)$ & \\
\hline Edoxaban & $62(4.6)$ & $30(4.4)$ & $32(4.8)$ & \\
\hline Aspirin & $301(17.8)$ & $140(16.8)$ & $161(18.7)$ & 0.309 \\
\hline Clopidogrel & $120(7.2)$ & $53(6.5)$ & $67(7.9)$ & 0.276 \\
\hline Statin & $646(35.9)$ & $324(36.0)$ & $322(35.8)$ & 0.922 \\
\hline ARB & $654(36.3)$ & $328(36.4)$ & $326(36.2)$ & 0.922 \\
\hline ACEI & $86(4.8)$ & $50(5.6)$ & $36(4.0)$ & 0.122 \\
\hline$\beta$-Blocker & & & & $0.027^{\mathrm{a}}$ \\
\hline Bisoprolol & $356(40.7)$ & $206(44.1)$ & $150(36.8)$ & \\
\hline Carvedilol & $246(28.1)$ & $133(28.5)$ & $113(27.7)$ & \\
\hline Metoprolol & $75(8.6)$ & $37(7.9)$ & $38(9 \cdot 3)$ & \\
\hline Nebivolol & $59(6.7)$ & $33(7.1)$ & $26(6.4)$ & \\
\hline Propranolol & $115(13.1)$ & $45(9.6)$ & $70(17.2)$ & \\
\hline Diltiazem & $107(23.4)$ & $61(24.4)$ & $46(22.2)$ & 0.910 \\
\hline Digoxin & $110(6.1)$ & $98(10.9)$ & $12(1.3)$ & $<0.001^{a}$ \\
\hline AFCA after enrollment & $119(6.6)$ & o & $119(13.2)$ & $<0.001^{a}$ \\
\hline \multicolumn{5}{|l|}{$\mathrm{AAD}$} \\
\hline Propafenone & & o & $181(20.1)$ & \\
\hline Flecainide & & o & $281(38.4)$ & \\
\hline Pilsicanide & & o & $27(3.7)$ & \\
\hline Dronedarone & & o & $46(6.3)$ & \\
\hline Amiodarone & & o & $141(9.2)$ & \\
\hline Sotalol & & o & $14(0.9)$ & \\
\hline
\end{tabular}

Values are presented as number (\%).

ARB, angiotensin receptor blocker; ACEI, angiotensin converting enzyme inhibitor; AFCA, atrial fibrillation catheter ablation; AAD, anti-arrhythmic drug.

${ }^{\text {a}}$ Statistically significant.

vs. $1.0 \%, p=0.011$ ), and the incidence rate of ischemic stroke in the rhythm control group was 0.7 per 1,000 person-years compared with 6.9 per 1,000 person-years in the rate control group shown in Fig. 1. Major bleeding and all-cause deaths were similar between all the patients treated with the rhythm and rate control strategies shown in Supplementary Figs. 2 and 3. In addition, all cause-hospitalization was similar between all the patients treated with the rhythm and rate control strategies shown in Table 4 and Supplementary Fig. 4.

\section{DISCUSSION}

In the prospective national AF cohort, a patient treated with the rhythm control strategy is associated with a lower risk of ischemic stroke occurrence for at least a 1-year follow-up compared to the rate control strategy.

In the present study, the overall incidence rate of stroke (7.2 per 1,000 person-years) was similar as previously reported $[24,25]$. After the propensity score matched analysis for comparison between these treatment strategies, the incidence rate of ischemic stroke 
(0.1\% vs. $1.0 \%$ ) in the rhythm and rate control groups was significantly lower than the previous Western registry, Registry on Cardiac Rhythm Disorders Assessing the Control of Atrial Fibrillation (RECORDAF; 1.7\% vs. 2.8\%) and Outcomes Registry for Better Informed Treat-

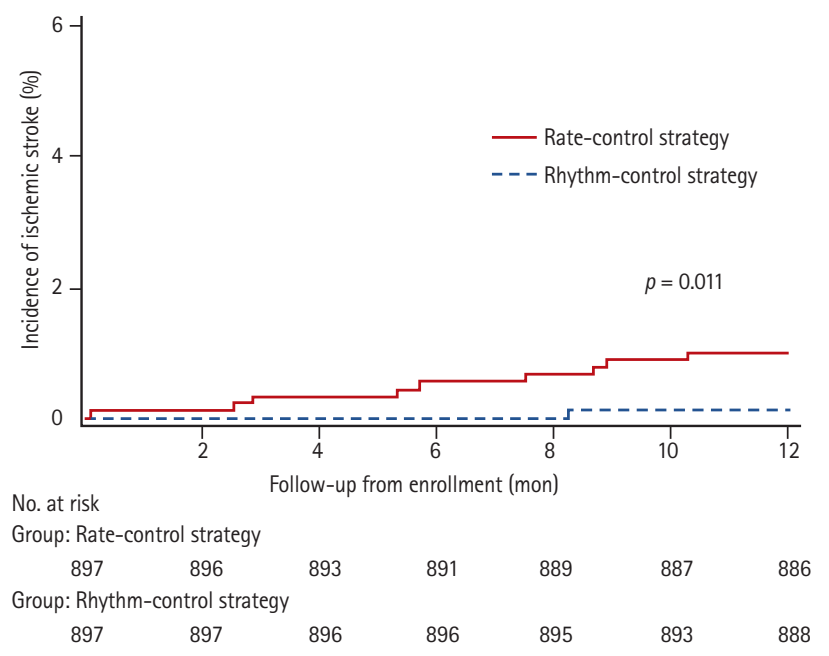

Figure 1. The comparison of the cumulative occurrence of ischemic stroke between the rhythm and rate control strategies (propensity score matching). ment of Atrial Fibrillation (ORBIT-AF; 2.5\% vs.3.2\%) at the 1-year follow-up [26,27]. Classic trials comparing the rate versus rhythm control strategy with only antiarrhythmic drugs such as the Atrial Fibrillation Follow-up Investigation of Rhythm Management (AFFIRM) trial, RAte Control versus Electrical cardioversion (RACE), and The Atrial Fibrillation and Congestive Heart Failure (AF-CHF) trial showed a non-statistically significant higher incidence of ischemic stroke in the rate control strategy, and recent comparisons of the differences between the rhythm and rate control strategy also showed that the occurrence of stroke seems to be similar with an overall odds ratio of 0.99 (95\% confidence interval [CI], 0.75 to 1.30) and a risk ratio (RR) of 0.96 (95\% CI, 0.68 to 1.34$)[9,28,29]$. In particular, the baseline characteristics in recent prospective cohorts including RECORDAF and ORBIT-AF had a greater proportion of advanced age, underlying cardiovascular diseases and a higher $\mathrm{CHADS}_{2}$ or $\mathrm{CHA}_{2} \mathrm{DS}_{2}$-VASC score than those in the present study [24]. These results have implicated that the rate of stroke occurrence could be more influenced by advanced age and underlying cardiovascular disor-

Table 4. After propensity score matching, comparison of clinical outcomes for the rhythm and the rate control strategy groups

\begin{tabular}{|c|c|c|c|c|}
\hline Variable & $\operatorname{ALL}(n=1,800)$ & Rate control $(n=900)$ & Rhythm control $(\mathrm{n}=900)$ & $p$ value \\
\hline Sinus rhythm at 1-yr follow-up & $1,156(64.2)$ & $413(45.8)$ & $743(82.5)$ & $<0.001^{a}$ \\
\hline Ischemic stroke & $10(0.6)$ & $9(1.0)$ & $1(0.1)$ & $0.011^{a}$ \\
\hline Hemorrhagic stroke & $4(0.3)$ & $1(0.1)$ & $3(0.4)$ & 0.274 \\
\hline All bleeding & $70(3.9)$ & $28(3.1)$ & $42(4.7)$ & 0.088 \\
\hline Major bleeding & $11(0.6)$ & $5(0.6)$ & $6(0.6)$ & 0.762 \\
\hline Death & $6(0.3)$ & $1(0.1)$ & $5(0.6)$ & 0.102 \\
\hline Hospitalization & $86(4 \cdot 7)$ & $38(4.2)$ & $48(5 \cdot 3)$ & 0.269 \\
\hline Heart failure aggravation & $13(0.7)$ & $7(0.7)$ & $6(0.7)$ & \\
\hline Bleeding control & $7(0.3)$ & $3(0.3)$ & $4(0.3)$ & \\
\hline Rate or rhythm control & $17(0.9)$ & $4(0.4)$ & $13(1.4)$ & \\
\hline Drug-induced bradycardia & $5(0.3)$ & $1(0.1)$ & $4(0.4)$ & \\
\hline Non-cardiac surgery & $10(0.5)$ & $7(0.7)$ & $3(0.3)$ & \\
\hline Pacemaker or defibrillator & $3(0.2)$ & $1(0.1)$ & $2(0.7)$ & \\
\hline All-caused Infection & $10(0.5)$ & $5(0.5)$ & $5(0.5)$ & \\
\hline Cancer management & $8(0.4)$ & $4(1.4)$ & $4(0.4)$ & \\
\hline Coronary intervention & $5(0.3)$ & $1(0.1)$ & $4(0.4)$ & \\
\hline Neurologic disorder & $8(0.4)$ & $5(0.5)$ & $3(0.3)$ & \\
\hline
\end{tabular}

Values are presented as number (\%).

${ }^{\text {a }}$ Statistically significant. 
ders than by the rhythm control strategy. In the recent Japanese registry, advanced age and a higher $\mathrm{CHA}_{2} \mathrm{DS}_{2}$ VASC score were also considered as independent risk factors for the occurrence of ischemic stroke [25].

The rhythm control strategy has been attributed to the inefficiency of antiarrhythmic drugs, and their adverse effects offset the beneficial effects to maintain a sinus rhythm [6]. In the present study, $66 \%$ of the rhythm control patients with class Ic anti-arrhythmic drugs and $34 \%$ of those with catheter ablation have been treated compared with the previous RECORDAF (overall $2 \%$ of those, ablation) and ORBIT-AF (overall $6 \%$ of those, ablation) registry to maintain a sinus rhythm during follow-up. Non-pharmacological catheter ablation could be also significantly associated with lower rates of stroke occurrence in patients with NVAF [7,8,14]. This beneficial effect of stroke prevention associated with catheter ablation has been demonstrated to maintain a sinus rhythm and to reduce the burden of AF compared with anti-arrhythmic drugs $[1,14,27]$. In the present study, the rhythm control group, which also enrolled $21.7 \%$ patients treated with catheter ablation, had more frequently maintained a sinus rhythm compared with the rate control group compared to previous studies [24,29]. The result of the lower risk of stroke occurrence in the rhythm control strategy is also consistent with that of other literature $[1,8]$ which is thus confirmatory in the prospective cohort registry. In addition, the better positive responses to the class Ic anti-arrhythmic drugs in NVAF patients without structural heart disease and ethnic differences might influence the maintenance of sinus rhythm compared with those enrolled from other countries [30].

OACs are the most important contributor to a lower risk of ischemic stroke [4,31], and direct OACs have a beneficial effect on additional significant reductions of overall $20 \%$ relative to conventional warfarin in the occurrence of stroke [5]. In the present study, a direct OAC was strongly recommended for patients treated with unsuccessful adjusted-dose warfarin therapy targeting an international normalized ratio (INR) of 2.0 to 3.0 or time in the therapeutic range (TTR) > 70\% [18] shown in Table 3. A direct OAC was more frequently and similarly prescribed between the patients in the rhythm control and rate control groups (72\% vs. $76 \%$ ) among the $74 \%$ OAC-indicated AF patients compared with the previous
RECORDAF (59\% OAC-indicated) and ORBIT-AF (72\% OAC-indicated) registry $[26,27]$.

The rhythm control strategy had a similar risk of OAC-related bleeding compared with that of the rate control strategy ( $4.7 \%$ vs. $3.1 \%, p=0.088)$ which is also concordant with a previous registry [27]. The rhythm control strategy offers no beneficial effect on all-cause hospitalization in patients with NVAF compared with the rate control strategy, and previous meta-analyses reported an overall RR of 0.95 (95\% CI, 0.76 to 1.19) and 1.15 (95\% CI, 0.15 to 1.88 ) $[9,10,28,32,33]$. The rhythm control strategy had no harmful effects in terms cardiovascular events, bleeding and mortality compared with the rate control strategy during the 1-year follow-up period shown in Table 4 and the Supplementary Figs. 1-4 $[9,12,28]$.

Although a comparison of the difference between the rate and rhythm control strategies has been recently released in randomized trials showing a similar effect for stroke prevention (catheter ablation versus antiarrhythmic drug therapy for atrial fibrillation [CABANA] [34]), the present study could be another piece of evidence of real practice that the rhythm control strategy has a beneficial effect on the prevention of stroke occurrence in selected NVAF patients with modest underlying diseases before advanced age.

Our study has several limitations. First, overall, $40 \%$ of all patients were clinically followed up during 1-year, and this could raise the generalizability of the results. Second, the propensity matching analysis could not prove the causality of a treatment strategy and an effect as the assignment of the randomization. Third, our study may not have a sufficient power for the association of ischemic stroke occurrence due to the low incidence of ischemic stroke. Fourth, patients had more persistent AF on rate control even after the matched analysis which introduces bias to include more advanced atrial disease. Fifth, no events occurred in patients treated with warfarin, and $25 \%$ of the enrolled patients were still treated with warfarin during the follow-up. Sixth, non-pharmacological procedures for rhythm control with a relapse were undertaken by selected patients and physicians, and monitoring for a relapse could also be limited to the subclinical AF and the poor correlation of AF symptoms. Therefore, the results may not be representative of a comparison between the rate and all the rhythm 
control strategies.

In conclusion, in this prospective national AF cohort with propensity score matching analysis, the rhythm control strategy showed a beneficial effect in terms of lowering the risk of ischemic stroke and no harmful effect in terms of hospitalization compared with the rate control strategy in the selected NVAF.

\section{KEY MESSAGE}

1. The rate of all bleeding and hospitalization were similarly between the rhythm and rate control group.

2. The incidence rate of ischemic stroke in the rhythm control group was significantly lower than in the rate control group.

\section{Conflict of interest}

No potential conflict of interest relevant to this article was reported.

\section{Acknowledgments}

This research was supported by a grant from the Korean Healthcare Technology R\&D project funded by the Ministry of Health and Welfare (HCI15C1200).

\section{REFERENCES}

1. Bunch TJ, Crandall BG, Weiss JP, et al. Patients treated with catheter ablation for atrial fibrillation have longterm rates of death, stroke, and dementia similar to patients without atrial fibrillation. J Cardiovasc Electrophysiol 2011;22:839-845.

2. Kirchhof P, Benussi S, Kotecha D, et al. 2016 ESC guidelines for the management of atrial fibrillation developed in collaboration with EACTS. Eur Heart J 2016;37:28932962.

3. January CT, Wann LS, Alpert JS, et al. 2014 AHA/ACC/HRS guideline for the management of patients with atrial fibrillation: a report of the American College of Cardiology/American Heart Association Task Force on practice guidelines and the Heart Rhythm Society. Circulation 2014;130:e199-e267.

4. Freedman B, Potpara TS, Lip GY. Stroke prevention in atrial fibrillation. Lancet 2016;388:806-817.

5. Ruff CT, Giugliano RP, Braunwald E, et al. Comparison of the efficacy and safety of new oral anticoagulants with warfarin in patients with atrial fibrillation: a meta-analysis of randomised trials. Lancet 2014;383:955-962.

6. Rolf S, Kornej J, Dagres N, Hindricks G. What can rhythm control therapy contribute to prognosis in atrial fibrillation? Heart 2015;101:842-846.

7. Bunch TJ, May HT, Bair TL, et al. Atrial fibrillation ablation patients have long-term stroke rates similar to patients without atrial fibrillation regardless of CHADS2 score. Heart Rhythm 2013;10:1272-1277.

8. Saliba W, Schliamser JE, Lavi I, Barnett-Griness O, Gronich N, Rennert G. Catheter ablation of atrial fibrillation is associated with reduced risk of stroke and mortality: a propensity score-matched analysis. Heart Rhythm 2017;14:635-642.

9. Al-Khatib SM, Allen LaPointe NM, Chatterjee R, et al. Rate- and rhythm-control therapies in patients with atrial fibrillation: a systematic review. Ann Intern Med 2014;160:760-773.

10. Wyse DG, Waldo AL, DiMarco JP, et al. A comparison of rate control and rhythm control in patients with atrial fibrillation. N Engl J Med 2002;347:1825-1833.

11. Scheinman MM. Atrial fibrillation therapy: rate versus rhythm control. Pacing Clin Electrophysiol 2003;26:11751177 .

12. Gillinov AM, Bagiella E, Moskowitz AJ, et al. Rate control versus rhythm control for atrial fibrillation after cardiac surgery. N Engl J Med 2016;374:1911-1921.

13. Ionescu-Ittu R, Abrahamowicz M, Jackevicius CA, et al. Comparative effectiveness of rhythm control vs rate control drug treatment effect on mortality in patients with atrial fibrillation. Arch Intern Med 2012;172:997-1004.

14. Tsadok MA, Jackevicius CA, Essebag V, et al. Rhythm versus rate control therapy and subsequent stroke or transient ischemic attack in patients with atrial fibrillation. Circulation 2012;126:2680-2687.

15. Kim H, Kim TH, Cha MJ, et al. A prospective survey of atrial fibrillation management for real-world guideline adherence: COmparison study of Drugs for symptom control and complication prEvention of Atrial Fibrillation (CODE-AF) Registry. Korean Circ J 2017;47:877-887.

16. Calkins H, Brugada J, Packer DL, et al. HRS/EHRA/ECAS expert Consensus Statement on catheter and surgical ablation of atrial fibrillation: recommendations for per- 
sonnel, policy, procedures and follow-up. A report of the Heart Rhythm Society (HRS) Task Force on catheter and surgical ablation of atrial fibrillation. Heart Rhythm 2007;4:816-861.

17. Hatano S. Experience from a multicentre stroke register: a preliminary report. Bull World Health Organ 1976;54:541-553.

18. Jung KH, Yu KH, Kim YD, et al. Antithrombotic management of patients with nonvalvular atrial fibrillation and ischemic stroke or transient ischemic attack: executive summary of the Korean clinical practice guidelines for stroke. J Stroke 2015;17:210-215.

19. Stevens PE, Levin A; Kidney Disease: Improving Global Outcomes Chronic Kidney Disease Guideline Development Work Group Members. Evaluation and management of chronic kidney disease: synopsis of the kidney disease: improving global outcomes 2012 clinical practice guideline. Ann Intern Med 2013;158:825-830.

20. January CT, Wann LS, Alpert JS, et al. 2014 AHA/ACC/HRS guideline for the management of patients with atrial fibrillation: a report of the American College of Cardiology/American Heart Association Task Force on Practice Guidelines and the Heart Rhythm Society. J Am Coll Cardiol 2014;64:e1-e76.

21. Schulman S, Kearon C; Subcommittee on Control of Anticoagulation of the Scientific and Standardization Committee of the International Society on Thrombosis and Haemostasis. Definition of major bleeding in clinical investigations of antihemostatic medicinal products in non-surgical patients. J Thromb Haemost 2005;3:692-694.

22. Kaatz S, Ahmad D, Spyropoulos AC, Schulman S; Subcommittee on Control of Anticoagulation. Definition of clinically relevant non-major bleeding in studies of anticoagulants in atrial fibrillation and venous thromboembolic disease in non-surgical patients: communication from the SSC of the ISTH. J Thromb Haemost 2015;13:2119-2126.

23. Austin PC. An introduction to propensity score methods for reducing the effects of confounding in observational studies. Multivariate Behav Res 2011;46:399-424.

24. Steinberg BA, Holmes DN, Ezekowitz MD, et al. Rate versus rhythm control for management of atrial fibrillation in clinical practice: results from the Outcomes Registry for Better Informed Treatment of Atrial Fibrillation (ORBIT-AF) registry. Am Heart J 2013;165:622-629.
25. Suzuki S, Yamashita T, Okumura K, et al. Incidence of ischemic stroke in Japanese patients with atrial fibrillation not receiving anticoagulation therapy: pooled analysis of the Shinken Database, J-RHYTHM Registry, and Fushimi AF Registry. Circ J 2015;79:432-438.

26. Noheria A, Shrader P, Piccini JP, et al. Rhythm control versus rate control and clinical outcomes in patients with atrial fibrillation: results from the ORBIT-AF registry. JACC Clin Electrophysiol 2016;2:221-229.

27. Camm AJ, Breithardt G, Crijns H, et al. Real-life observations of clinical outcomes with rhythm- and rate-control therapies for atrial fibrillation RECORDAF (Registry on Cardiac Rhythm Disorders Assessing the Control of Atrial Fibrillation). J Am Coll Cardiol 2011;58:493-501.

28. Chatterjee S, Sardar P, Lichstein E, Mukherjee D, Aikat S. Pharmacologic rate versus rhythm-control strategies in atrial fibrillation: an updated comprehensive review and meta-analysis. Pacing Clin Electrophysiol 2013;36:122-133.

29. Sethi NJ, Feinberg J, Nielsen EE, Safi S, Gluud C, Jakobsen JC. The effects of rhythm control strategies versus rate control strategies for atrial fibrillation and atrial flutter: a systematic review with meta-analysis and Trial Sequential Analysis. PLoS One 2017;12:e0186856.

30. Meinertz T, Lip GY, Lombardi F, et al. Efficacy and safety of propafenone sustained release in the prophylaxis of symptomatic paroxysmal atrial fibrillation (The European Rythmol/Rytmonorm Atrial Fibrillation Trial [ERAFT] Study). Am J Cardiol 2002;90:1300-1306.

31. Sherman DG. Stroke prevention in atrial fibrillation: pharmacological rate versus rhythm control. Stroke 2007;38(2 Suppl):615-617.

32. Hohnloser SH, Kuck KH, Lilienthal J. Rhythm or rate control in atrial fibrillation: pharmacological Intervention in Atrial Fibrillation (PIAF): a randomised trial. Lancet 2000;356:1789-1794.

33. Hagens VE, Vermeulen KM, TenVergert EM, et al. Rate control is more cost-effective than rhythm control for patients with persistent atrial fibrillation: results from the RAte Control versus Electrical cardioversion (RACE) study. Eur Heart J 2004;25:1542-1549.

34. Packer DL, Mark DB, Robb RA, et al. Catheter ablation versus antiarrhythmic drug therapy for atrial fibrillation (CABANA) trial: study rationale and design. Am Heart J 2018;199:192-199. 
Supplementary Table 1. Comparison of the baseline characteristics of the total patients with loss of follow-up and with over 1-year follow-up

\begin{tabular}{|c|c|c|c|c|}
\hline Characteristic & $\operatorname{ALL}(\mathrm{n}=6,280)$ & Loss of follow-up $(n=3,767)$ & Over 1-yr follow-up $(\mathrm{n}=2,513)$ & $p$ value \\
\hline Age, yr & $67 \pm 10$ & $67 \pm 10$ & $67 \pm 10$ & 0.675 \\
\hline Male sex & $3,966(63.2)$ & $2,392(63.5)$ & $1,553(61.8)$ & 0.543 \\
\hline $\mathrm{BMI}, \mathrm{kg} / \mathrm{m}^{2}$ & $24.6 \pm 3.4$ & $24 \cdot 5 \pm 3 \cdot 3$ & $24 \cdot 3 \pm 3.1$ & 0.224 \\
\hline Hypertension & $4,275(67.5)$ & $2,516(64 \cdot 9)$ & $1,759(70.0)$ & 0.176 \\
\hline Diabetes mellitus & $1,607(25.4)$ & $928(24 \cdot 5)$ & $679(27.0)$ & 0.057 \\
\hline Dyslipidemia & $2,209(34.8)$ & $1,335(35 \cdot 4)$ & $874(34.8)$ & 0.313 \\
\hline Previous MI & $213(3.2)$ & $107(3.0)$ & $106(4.2)$ & 0.054 \\
\hline Previous HF & $596(12.5)$ & $355(9.7)$ & $241(9.6)$ & 0.051 \\
\hline Previous CKD & $312(10.0)$ & $78(9.4)$ & $234(9 \cdot 3)$ & 0.603 \\
\hline Previous cancer & $631(10.0)$ & $393(10.4)$ & $238(9.5)$ & 0.137 \\
\hline Previous stroke & $928(14.7)$ & $520(13.8)$ & $408(16.2)$ & 0.051 \\
\hline Previous ECV & $1,004(15 \cdot 9)$ & $558(14.8)$ & $446(17.7)$ & $<0.001^{\mathrm{a}}$ \\
\hline Previous AFCA & $956(15 \cdot 2)$ & $534(14.1)$ & $422(16.8)$ & $<0.001^{\mathrm{a}}$ \\
\hline $\mathrm{CHA}_{2} \mathrm{DS}_{2}$-VASC score & $2.6 \pm 1.6$ & $2.6 \pm 1.6$ & $2.7 \pm 1.6$ & 0.896 \\
\hline HAS-BLED score & $1.8 \pm 1.0$ & $1.8 \pm 1.0$ & $1.8 \pm 1.0$ & 0.834 \\
\hline Systolic BP, mmHg & $122 \pm 15$ & $122 \pm 15$ & $121 \pm 15$ & 0.524 \\
\hline Diastolic BP, mmHg & $75 \pm 11$ & $75 \pm 11$ & $73 \pm 11$ & 0.154 \\
\hline Heart rate, bpm & $75 \pm 15$ & $76 \pm 16$ & $74 \pm 15$ & 0.138 \\
\hline LA size, $\mathrm{mm}$ & $41 \pm 7$ & $41 \pm 7$ & $41 \pm 7$ & 0.896 \\
\hline Ejection fraction, \% & $61 \pm 7$ & $61 \pm 7$ & $61 \pm 7$ & 0.826 \\
\hline Baseline AF & $1,690(26.9)$ & $520(13.8)$ & $1,170(46.5)$ & $<0.001^{a}$ \\
\hline Symptomatic AF & $3,206(51.0)$ & $2,151(57 \cdot 1)$ & $1,055(57.8)$ & 0.507 \\
\hline AF type & & & & 0.328 \\
\hline Paroxysmal AF & $3,441(54.8)$ & $2,419(64.2)$ & $1,055(63.3)$ & \\
\hline Persistent AF & $2,639(42.0)$ & $1,189(31.5)$ & $1,450(30.7)$ & \\
\hline Permanent AF & $107(1.7)$ & $99(2.6)$ & $8(5.8)$ & \\
\hline
\end{tabular}

Values are presented as mean \pm standard deviation or number (\%).

BMI, body mass index; MI, myocardial infarction; HF, heart failure; CKD, chronic kidney disease; ECV, electrical cardioversion; AFCA, atrial fibrillation catheter ablation; HAS-BLED, Hypertension, Abnormal renal/liver function, Stroke, Bleeding history or predisposition, Labile international normalized ratio, Elderly, and Drugs/alcohol concomitantly; BP, blood pressure; LA, left atrium; AF, atrial fibrillation.

${ }^{a}$ Statistically significant. 


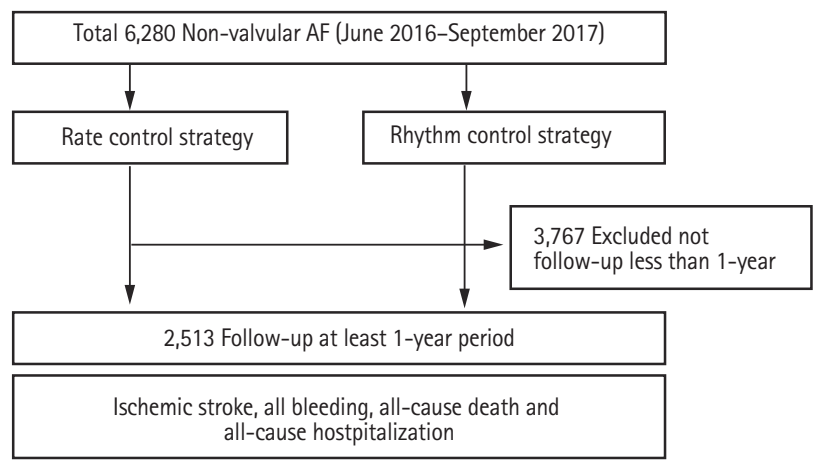

Supplementary Figure 1. Study flow chart. AF, atrial fibrillation. 


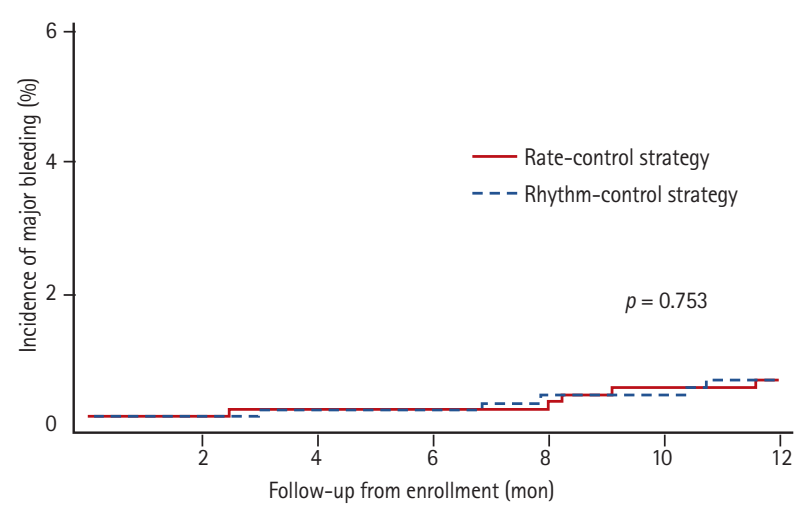

No. at risk

Group: Rate-control strategy

$\begin{array}{ccccccc}897 & 893 & 889 & 886 & 882 & 875 & 870 \\ \text { Group: Rhythm-control strategy } & & & & & \\ 897 & 891 & 889 & 883 & 875 & 869 & 859\end{array}$

Supplementary Figure 2. Comparison of the cumulative occurrence of major bleeding between the rhythm and rate control strategies. 


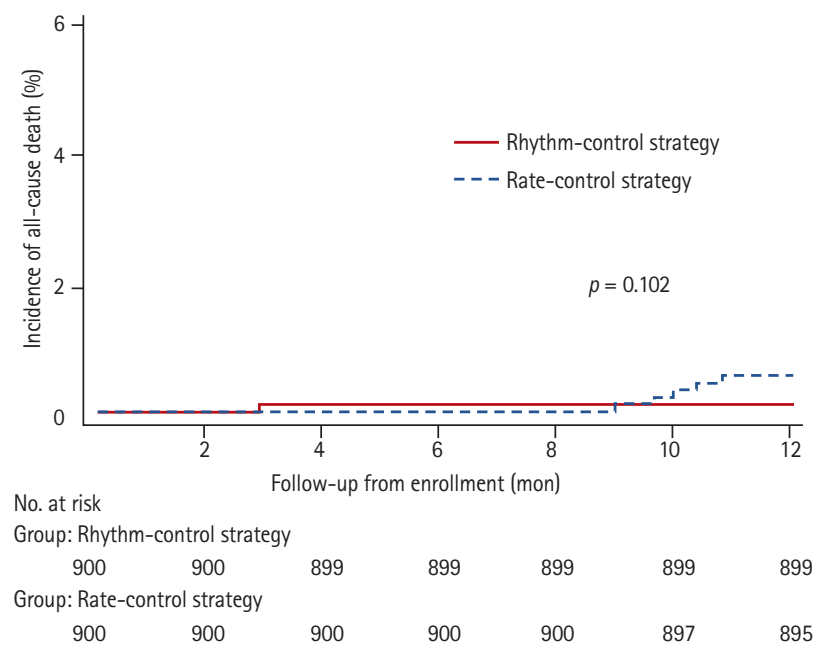

Supplementary Figure 3. Comparison of the cumulative occurrence of all-cause death between the rhythm and rate control strategies. 




No. at risk

Group: Rhythm-control strategy

$\begin{array}{ccccccc}900 & 857 & 824 & 793 & 782 & 761 & 744 \\ \text { Group: Rate-control strategy } & & & & & \\ 900 & 839 & 798 & 744 & 716 & 680 & 656\end{array}$

Supplementary Figure 4. Comparison of the cumulative occurrence of all-cause hospitalization between the rhythm and rate control strategies. 